\title{
DESIGN OF CLUSTERING ANALYZER BASED ON SYSTOLIC ARRAY ARCHITECTURE
}

\author{
Mao-Fu Lai \\ Dept. of Electrical Engineering \\ National Taiwan University \\ Taipei, Taiwan, R.O.C.
}

Yan-Pei Wu

Dept. of Electrical Engineering

National Taiwan University

Taipei, Taiwan, R.O.C.
Chaur-Heh Hsieh

Dept. of Electrical Engineering

Chang-Cheng Institute of Technology

Tashi, Taiwan, R.O.C.

\section{ABSTRACT}

Clustering analysis is an important technique in image processing and unsupervised pattern recognition. The squared-error clustering algorithm is the most well-known method in clustering analysis. Recent progress in VLSI technology stimulates the concept to implement the squared-error clustering algorithm by hardware for high speed applications. This paper presents a systolic architecture for the squared-error clustering algorithm. The proposed architecture exploits a 2-dimensional systolic array which uses intensively parallel and pipelined processing. The architecture dramatically reduces the huge number of processing elements which are required by other previous architectures. Furthermore, the same organization can be utilized for applications where the number of input patterns is varied. In addition, the time complexity of our architecture is reduced in comparison with earlier architectures. The cost-effective VLSI implementation for high speed clustering analysis can be realized with considerately less circuit complexity based on this novel architecture.

\section{1.. INTRODUCTION}

Clustering analysis is a valuable technique in data analysis, especially when limited information about the data is known [1]. The resuits of clustering analysis are used to find natural grouping in a data set; this helps the user understand the structure of the data. Applications of clustering techniques include image segmentation, image registration, speech recognition, pattern recognition, etc. [241.

Numerous algorithms have been developed for clustering analysis [5-6]. The most well-known clustering technique for application in image processing and pattern recognition is the squared-error clustering method. Recently, the advance in VLSI technology has brought about the possibility of implementing the clustering technique directly by hardware chips. However, huge quantities of circuits are still required to implement the squarederror clustering algorithm, which makes the VLSI implementation very difficult. Therefore, it is essential that a good architecture be available to reduce both computational and circuit complexities. --

Several VLSI architectures for clustering analysis based on the squared error clustering algorithm were presented before [7-9]. A systolic array architecture using two-level pipelined design was proposed in [7]. The one pass time complexity is $O(N x \max (M, K))$, where $\mathrm{N}$ is the total number of input pattems, $K$ is the total number of clusters, $M$ is the dimension of the input vector. It can be seen that the required execution time is very long since $N$ is a large number in most clustering applications. Another VLSI architecture which reduces one pass time complexity from $O(N \mathrm{xmax}(M, K))$ to $O(N+M+K)$ was proposed by Cheng and Tong in [9]. This architecture consists of four Modules and the number of PEs in Modules A, C, and D depends on $N$ : Module A consists of $N \times K$ processing elements (PEs); Module C, $N \mathrm{x} M$ shift registers and $2(N$ 1) delay registers; and Module D, N PEs [9]. Since N is a large number in practical applications, a tremendous amount of PEs are required in Modules A, C, and D according to the architecture proposed by Cheng and Tong. Moreover, all $N$ patterns appear at the system inputs in parallel, which is not practical for VLSI implementation.

A novel VLSI architecture for a clustering analyzer is proposed in this paper. Modules A, B, C, and D are used in accordance with the organization in [9] in order to make a direct comparison. The hardware complexity of the proposed architecture is decreased to $M \times K X$ PEs for Module A, to approximately $M$ $\left(\frac{3}{2} M+2 \sqrt{K}-\frac{1}{2}\right)$ registers for Module $\mathrm{C}$, and to $K$ PEs for Module D. Moreover, the one pass time complexity is reduced to approximately $O(N+M+3 \sqrt{K})$, and thus the reduction in computation time of the proposed architecture is significant when $K$ is large.

In this paper a systolic VLSI architecture for the squared-error clustering algorithm is presented. The notations are first briefly explained, and system flowchart for the squared-error clustering algorithm is described. Next, system operation and organization are introduced. Then, the architecture and configuration for each module are formulated. Finally, the hardware and computational complexities for the proposed architecture are compared with the results of other previous architectures.

\section{NOTATIONS}

The notations frequently used in squared-error clustering techniques are explained as follows.

1) $N$ : the number of input patterns for a data set to be partitioned into clusters.

2) $M$ : the dimension of features of each input pattern.

3) $P$ : An $N \times M$ pattern matrix including $N$ vectors with $M$ features each.

4) $P(i)$ : a $1 \times M$ vector for the ith input pattern, where $1 \leq i \leq N$.

5) $p(i, m)$ : an element in the matrix $P(N \times M)$, representing the $m$ th feature of the th input pattern, where $1 \leq i \leq N, 1 \leq m \leq M$.

6) $K$ : the total number of clusters. 
7) C: a $K \mathrm{x} M$ cluster center matrix including $K$ vectors with $M$ features each.

8) $C(k)$ : a $1 \times M$ vector for the $k$ th cluster center, where $1 \leq k \leq K$.

9) $c(k, m)$ : an element in the matrix $C(K \times M)$, representing the $m$ th feature of the $k$ th cluster center, where $1 \leq k \leq K, 1 \leq m \leq N$.

10) $L(i)$ : the label assigned to the ith pattern $P(i) . L(i)$ indicates the cluster number which the ith pattern belongs to, where $1 \leq L(i) \leq K, 1 \leq i \leq N$.

11) $S(k)$ : a set of input patterns whose labels $L(i)$ 's are equal to $k$.

$$
S(k)=\{i \mid L(i)=k \text {, for } 1 \leq i \leq N\}
$$

12) $|S(k)|:$ the total number of input patterns belonging to the cluster $k$, where $1 \leq k \leq K$.

13) $D_{i}^{k}$ : The squared Euclidean distance between the ith input pattern and the $k$ th cluster center. $D_{i}^{k}$ is defined as

$$
D_{i}^{k}=\sum_{m=1}^{M}[p(i, m)-c(k, m)]^{2}
$$

14) $\min \left(D_{j}\right)$

The smallest value of the squared distances for the ith pattern computed from (2).

15) $E(k)$ : The squared-error for the $k$ th cluster, defined as

$$
E(k)=\sum_{i \in S(k)} \min \left(D_{i}\right), \text { where } i \leq k \leq K
$$

16) $L(i)$ : An appropriate label $L(i)$ for the pattern $P(i)$. The nearest centroid clustering assignment used to find $L(i)$ is given by

$L(i)=k$ if $D_{i}^{j}<D_{i}^{k}$, for $k \neq j$, where $1 \leq j, k \leq K$

The calculation of new cluster centers is based on the following formula:

$$
c(k, m)=\frac{1}{|S(k)|} \sum_{i \in S(k)} p(i, m), \text { where } 1 \leq k \leq K, 1 \leq m \leq M
$$

\section{SYSTEM OPERATION AND ORGANIZATION}

The operation of clustering technique repeats labeling process and cluster center updating process until convergence is achieved [11]. Due to repeated computations of the squared-error clustering technique, a large number of arithmetic operations (including addition, subtraction, squaring, and magnitude comparison) are required even for a moderate number of input patterns. This demands substantial computation time to execute the squared-error clustering álgorithm. For real-time applications of clustering analysis, VLSI implementation is very attractive for the realization of the algorithm. The systolic array using high degree of parallel processing and pipelining is helpful to enhance the execution speed [11-15].

The system organization of the proposed VLSI design for the squared-error clustering algorithm is illustrated in Fig. 1, which is composed of four modules (Module A, B, C, D). Assume the input pattern matrix $P(N \mathrm{x} M)$ has been stored in memory, so data can be moved from memory to Module $\mathrm{C}$ when operation starts. Module $\mathrm{C}$ will arrange input data flow to Module $A$ and Module $B$ in an appropriate timing sequence. Input pattern $p(1,1)$ is applied to Module $\mathrm{A}$ at the first clock; $p(2,1)$ and $p(1,2)$ to Module $\mathrm{A}$ at the 2nd clock; $p(3,1), p(2,2)$ and $p(1,3)$, to Module $A$ at the 3 rd clock, etc. Module B receives $p(1,1) \ldots p(1, M)$ simultaneously from Module $\mathrm{C}$ at the instant when $L(1)$ is generated from Module $\mathrm{A}$.

Module A obtains the skewed input patterns from Module C, and calculates the squared Euclidean distance $D_{i}^{k}$ between every input pattern vector $P(i)$ and each cluster center vector $C(k)$. Module A generates the minimum squared distance $\min \left(D_{i}\right)$ and the corresponding label $L(i)$ for each input pattern sequentially at every clock cycle. $L(i)$ is fed to Module B and Module D for decoding, and $\min \left(D_{i}\right)$ is applied to Module $\mathrm{D}$ for calculation of the squarederror $E(k)$ for each cluster.

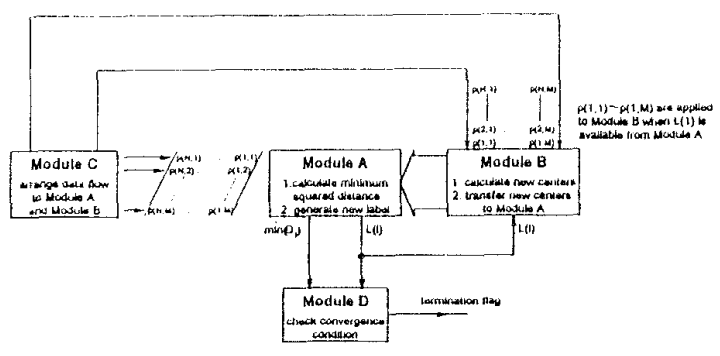

Fig. 1 System organization

Module B obtains data stream in vector-parallel pattern-serial format from Module $\mathrm{C}$. The pattern vector $P(i)$ is applied to the corresponding cluster assigned by $L(i)$ for the computation of the partial sum based on the formula in (5). After the last label $L(N)$ appears, new cluster centers will then be calculated in Module B based on (5). The divisions by $|S(k)|$ can be processed in parallel. After calculation of the new cluster centers is completed, the new cluster vector $C(k)$ is transferred to the corresponding registers in Module A by pipelined shift operation.

Module $\mathrm{D}$ receives $\min \left(D_{\mathrm{j}}\right)$ and $L(i)$ for each input pattern and computes the squared-error $E(k)$ for each cluster according to (3).

\section{MODULE A ARCHITECTURE}

The organization of Module A is illustrated in Fig. 2 (a). The total number of processing units (PUs) is $K$. The PUs are arranged in a 2-dimensional array so that parallel processing can be executed for the PUs located on the same dashed line. Also pipelined processing is performed for the PUs located on the same column; this can further speed up the squared distance computation. The arrangement of PUs is divided into $K=X \times Y$ with $X=Y$ if $K$ is the square of an integer number. If $K$ cannot be expressed as the square of an integer, then unequal $X$ and $Y$ which satisfy $X \mathbf{x} Y=K$ can be chosen, where $X$ and $Y$ are selected as near as possible.

The skewed array of $N$ input pattern vectors are applied in the horizontal direction to $\mathrm{PU}_{11}$. Input pattern $p(1,1)$ is applied to 
$\mathrm{PU}_{11}$ at the first clock, and the squared distance $D_{1}^{1}$ is generated at the $M$ th clock. Input pattern $p(1,1)$, after the delay of one clock cycle, is passed to $\mathrm{PU}_{12}$ and $\mathrm{PU}_{21}$ simultaneously at the 2nd clock. At the 3rd clock, $p(1,1)$ is passed to $\mathrm{PU}_{31}, \mathrm{PU}_{22}$, and $\mathrm{PU}_{13}$ for parallel processing to compute the squared distances of the first pattern concurrently.

The structure of each PU for Module A is shown in Fig. 2(b); it consists of $M$ processing elements (PEs) and a cell C. $\mathrm{PE}_{1} \ldots \mathrm{PE}_{M}$ are arranged vertically in a pipeline at $\mathrm{PE}_{j}$. The squared distance $D_{i}^{k}$, obtained from the output of $\mathrm{PE}_{M}$, is fed into the cell $\mathrm{C}$ for comparison with $\min \left(D_{i}\right)_{\text {in }}$ passed from the left $\mathrm{PU}$, and the smaller of the two will be passed to the right adjacent cell $\mathrm{C}$.

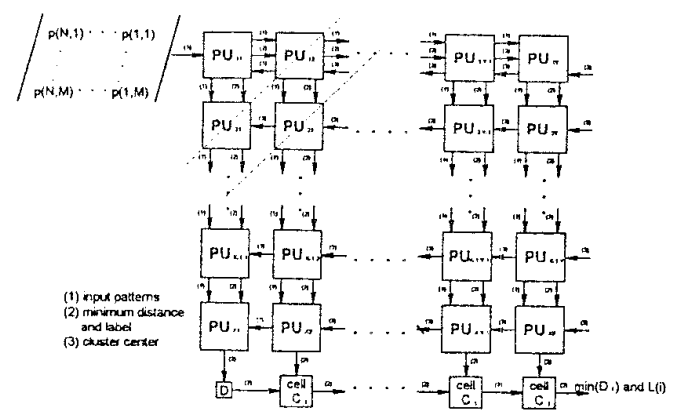

(a)

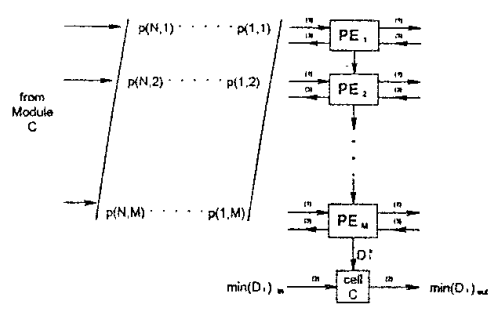

(b)
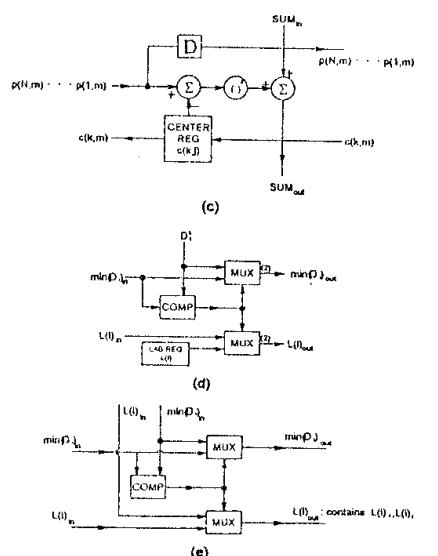

Fig. 2 Architecture of Module A

(a) System organization (b) Structure of PU (c) Internal structure of PU (d) Internal structure of Cell C (e) Internal structure of Cell $C_{1}$
The internal structure of each PE is shown in Fig. 2(c). Each PE performs the subtraction of $p(i, m)$ from $c(k, m)$, squares the difference, adds the result to the partially computed squared distance $\left(\mathrm{SUM}_{\mathrm{in}}\right)$ passed from the upper $\mathrm{PE}$, and passes the adder output $\left(\mathrm{SUM}_{\text {out }}\right)$ to the lower adjacent PE for further computation. The input pattern, after passing through the delay register $(D)$, is applied to the right adjacent PE. The input and output paths for center register are used for new center movement during cluster center updating.

The internal structure of cell $\mathrm{C}$ is shown in Fig. 2(d). A comparison operation is performed on the two inputs, $D_{i}^{k}$ from $\mathrm{PE}_{M}$ and $\min \left(D_{i}\right)_{\text {in }}$ from the left $\mathrm{PU}$, and the comparator output is used as the select line for the two MUXs. The smaller of two inputs, $\min \left(D_{i}\right)_{\text {out }}$, and the associated label, $L(i)_{\text {out }}$, will be passed to the output. Each label $L(i)$ of the label register in cell $\mathrm{C}$ is represented by a 2-dimensional code $L(i)_{x}$ and $L(i)_{y}$, which corresponds to the position of $\mathrm{PU}$ in the array. The 2-dimensional labels are utilized for 2-dimensional decoding in Module B and Module D. $L(i)_{\text {in }}$ and $L(i)_{\text {out }}$ in Fig. 2(d) are also represented in the same 2-dimensional format, though it is not indicated in the figure for simplicity.

Refer to the PUs in the bottom row of Module A in Fig. 2(a); the squared distance output of PUX1 appears one clock earlier than that of PUx2, therefore a delay register $(D)$ is required to delay one clock cycle of the output from the PUx1 for comparison with the minimum squared distance obtained in the next clock cycle from PUx2 output. The leftmost Cell $\mathrm{Cl}$ is used to compare delay register output with $\mathrm{PU}_{\mathrm{x} 2}$ output; the smaller distance $\left(\min \left(D_{i}\right)_{\text {out }}\right)$ and its associated label $\left(L(i)_{\text {out }}\right)$ are passed to the next adjacent $\mathrm{Cl}$ cell. The internal structure of cell $\mathrm{Cl}$ is shown in Fig. 2(e).

A complete systolic architecture of Module $A$ is illustrated in Fig. 3 for the example of nine clusters $(K=X \times X Y=3 \times 3)$ and three features $(M=3)$. The inputs of cell $\mathrm{C}$ in $\mathrm{PU}_{11}$ are connected to Dmax (the maximum distortion measure) and $\mathrm{L}_{d}$ (dummy label). Input patterns are pumped regularly in both horizontal direction (in the top row) and vertical direction (in all columns) across the systolic array, and are effectively used in all PEs.

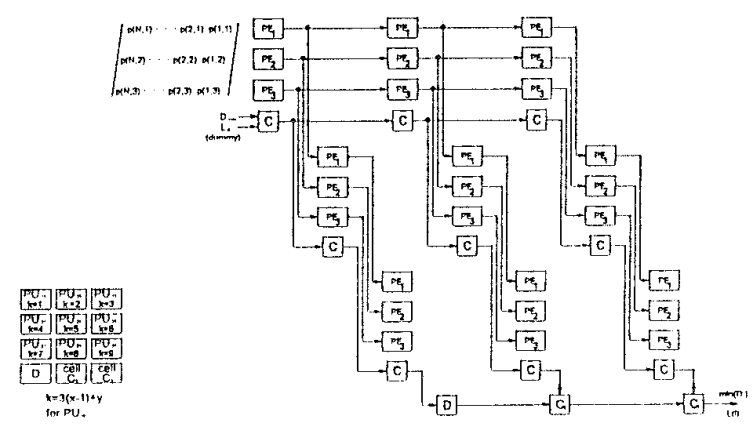

Fig. 3 Architecture of Module A with 9 clusters 
Fig. 4 illustrates the data flow and the comparison results in each clock cycle of the architecture in Fig. 3. The nine label registers and center registers of nine $(3 \times 3=9)$ PUs store the labels and clusier centers according to $k=3(x-1)+y$ for PUxy. Input patterns are first applied to the $P E s$ of $\mathrm{PU}_{11}$, and are passed to $\mathrm{PU}_{21}$ and $\mathrm{PU}_{12}$ one clock later. Assume $p(1, m)$ arrives at $\mathrm{PE}_{m}$ of $\mathrm{PU}_{11}$ at the $m$ th clock. $D_{1}^{1}, D_{2}^{1}, D_{3}^{1}$, and $D_{4}^{1}$ appear at the output of cell $\mathrm{C}$ in $\mathrm{PU}_{11}$ at the 4 th $(M+1=4)$, 5th $(M+2=5)$, 6th $(M+3=6)$ and 7 th $(M+4=7)$ clocks, respectively. At the 5th clock, cell $\mathrm{C}$ of $\mathrm{PU}_{12}$ and $\mathrm{PU}_{21}$ will generate $\min _{k=1,2}\left(D_{1}^{k}\right)$ and $\min _{k=1,4}\left(D_{1}^{k}\right)$ respectively. The minimum squared distance of the first pattern appears at the 9th $(M+X+Y=3+3+3=9)$ clock, and that of the 2 nd pattern appears at the 10 th $(M+X+Y+1=3+3+3+1=10)$ clock, etc. The first label $L(1)$ and the minimum distance $\min \left(D_{1}\right)$ for $P(1)$ are generated at the instant of the $(M+X+Y)$ th clock, and the last label $L(N)$ with the associated minimum distance $\min \left(D_{N}\right)$ for $P(N)$ is generated at the $(N+M+X+Y-1)$ th clock. If $X=Y=\sqrt{K}$, the time required to obtain the labels for all $N$ patterns is $(N+M+2 \sqrt{K}-1)$ clock cycles.

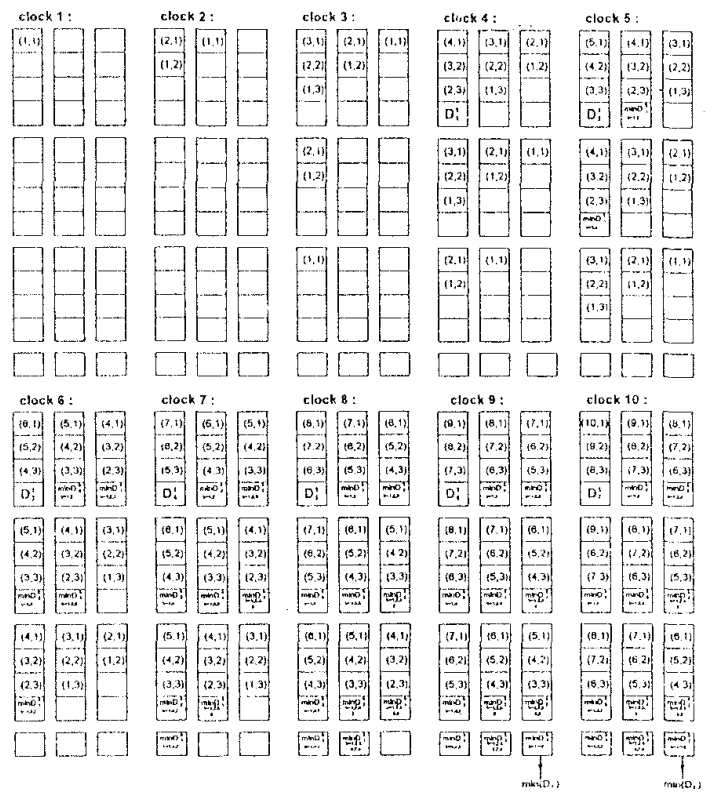

Fig. 4 Data stream in Module A with 9 clusters

\section{MODULE B ARCHITECTURE}

The organization of Module B is illustrated in Fig. 5(a); it is composed of $K$ PUs arranged in 2-dimensional arrays identical to Module A. There are two decoders to perform 2-dimensional decoder. The label $L(i)_{x}$ and $L(i)_{y}$ from Module $A$ are applied to $X$ decoder and $Y$ decoder respectively.

The functions of each PU in Module B involve the calculation of new cluster centers and the transfer of cluster centers to Module
A. The process of calculating new centers starts at the $(M+X+h)$ th clock, when the first label $L(1)$ is available from Module A. At this instant the first pattern vector $P(1)$ should be applied to the Module $\mathrm{B}$ input. Assuming that the new label for $L(1)$ is $k$, then $L(1)$ for the first pattern is decoded by $\mathrm{X}$ decoder and $\mathrm{Y}$ decoder to select the $\mathrm{PU}_{x y}$ corresponding to the $k$ th cluster, and the pattern vector $P(1)$ is applied to the $k$ th cluster center. This process repeats for all $\mathrm{N}$ patterns

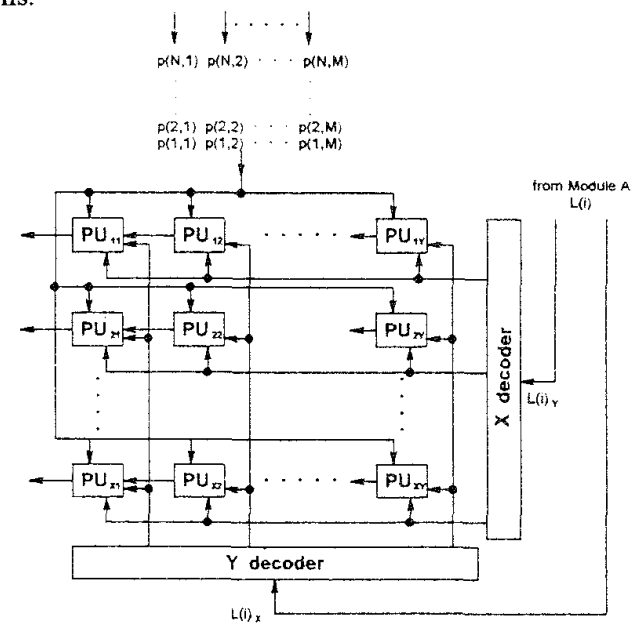

(a)

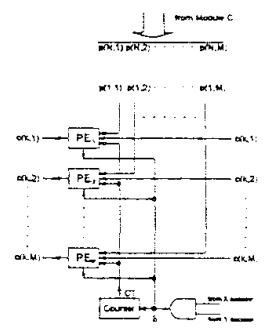

(b)

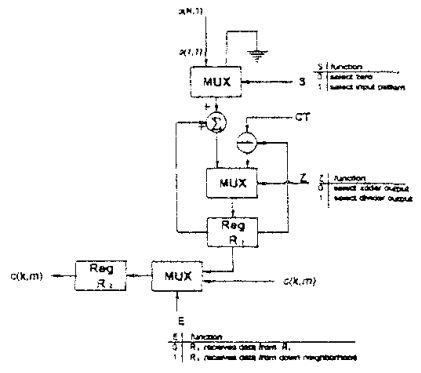

(c)
Fig. 5 Architecture of Module B

(a) System organization (b) Structure of PU (c) Internal structure for PE

The structure of a PU in Module B is shown in Fig. 5(b). The AND gate output, $S$, is used to select the pattern input $P(i)$ to all PEs when the PU is enabled $(S=1)$. $S$ signal also increments the counter which keeps track of the total number of input patterns assigned to this particular cluster so far. The internal structure of a PE is shown in Fig. 5(c). After $L(N)$ appears at the $(N+M+X+Y-1)$ th clock, all pattern vectors $P(1) \ldots P(N)$ have been added to the R1 registers in PEs of the associated PUs. Assume that it takes one clock cycle to complete the divisions for all clusters, then all new cluster centers will appear in all $\mathrm{R} 1$ registers at the $(N+M+X+Y)$ th clock. These new cluster centers should be moved to the center registers in Module $\mathrm{A}$. The movement is performed by cascading $\mathrm{R} 2$ registers through multiplexers. With $E=0$, the data in $\mathrm{R} 1$ will be transferred 
to $\mathrm{R} 2$ at the $(N+M+X+Y+1)$ th clock, and the next step is to shift the data in $\mathrm{R} 2$ of $\mathrm{PE}_{j}$ in the $\mathrm{PU}_{x y}$ of Module $\mathrm{B}$ to the corresponding center register of $\mathrm{PE} j$ in the $\mathrm{PU}_{x y}$ of Module $\mathrm{A}$. In conclusion, the one pass operation in Module $B$ is completed at the $(N+M+2 X+Y+1)$ th clock. If $X=Y=\sqrt{K}$, the total time required for one pass operation in Module $\mathrm{B}$ is $N+M+3 \sqrt{K}+1$.

\section{MODULE C ARCHITECTURE}

The organization of Module $\mathrm{C}$ is illustrated in Fig. 6. Once presented with an address from the Address Generator, each bank sends out an element for input pattern. The $M$ elements from all banks form a pattern vector. The delay registers in Fig. 6, with a total number of $\frac{M}{2}(M-1)$, are utilized to perform this function. Module $C$ should also send a data stream of input patterns to Module $\mathrm{B}$ at the instant starting from the $(M+X+Y)$ th clock. The serial-in serial-out (SISO) registers are employed to generate a delay of $(M+X+Y)$ clocks for all input patterns so that the first pattern $P(1)$ reaches Module $\mathrm{B}$ input at the $(M+X+Y)$ th clock and $P(N)$ arrives at Module $\mathrm{B}$ at the $(N+M+X+Y-1)$ th clock. Each SISO register further consists of $(M+X+Y)$ registers, so the total number of registers used in $M$ SISO registers is $M(M+X+Y)$. If $X=Y=\sqrt{K}$, the total number of registers in Module $\mathrm{C}$, (including delay registers and SISO registers) is $M(M+2 \sqrt{K})+\frac{M}{2}(M-1)=M\left(\frac{3}{2} M+2 \sqrt{K}-\frac{1}{2}\right)$

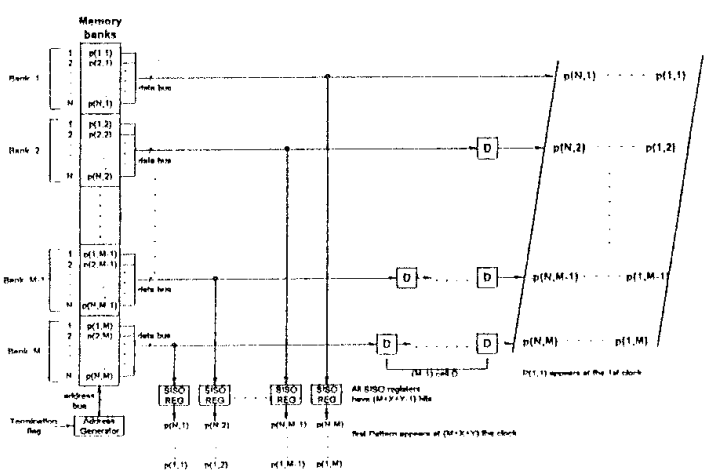

Fig. 6 Architecture of Module $\mathrm{C}$

\section{MODULE D ARCHITECTURE}

The organization of Module D is illustrated in Fig. 7(a). The PUs in Module $D$ are also arranged in a 2-dimensional array $(X \mathbf{x} Y=K)$ identical to Module A. Each PU is used to calculate the squared-error of the $k$ th cluster according to (3), and to check whether the $E(k) \leq$ THD condition is met.

The labels $L(i)_{x}$ and $L(i)_{y}$ from Module $\mathrm{A}$ are connected to $\mathrm{X}$ decoder and $\mathrm{Y}$ decoder of Module $\mathrm{D}$ to select one PU from the array in Fig. 7(a). When the PU corresponding to $L(i)$ is selected, the $\min \left(D_{j}\right)$ from Module $\mathrm{A}$ is applied to the input of the selected $\mathrm{PU}$, so that a partial sum of the squared-error for the $k$ th cluster is computed. The internal structure of each PU in Module D is shown in Fig. 7(b). Each PU consists of two registers, one adder, one comparator, and two AND gates. $\mathrm{Rl}$ is used to accumulate the partial sum of $\sum \min \left(D_{i}\right)$ from Module A. When $L(N)$ is generated from Module A at the $(N+M+X+Y-1)$ th clock, R1 in the PU for the $k$ th cluster will obtain the squared- error $E(k)$. The next step is to compare the content of $\mathrm{R} 1$ with the preloaded limit (THD) stored in R2. All PEs can perform the comparison simultaneously, and it takes one clock cycle to obtain the comparison results for all clusters. From the above description, a complete one pass operation in Module $\mathrm{D}$ requires $(N+M+X+Y+1)$ clocks.

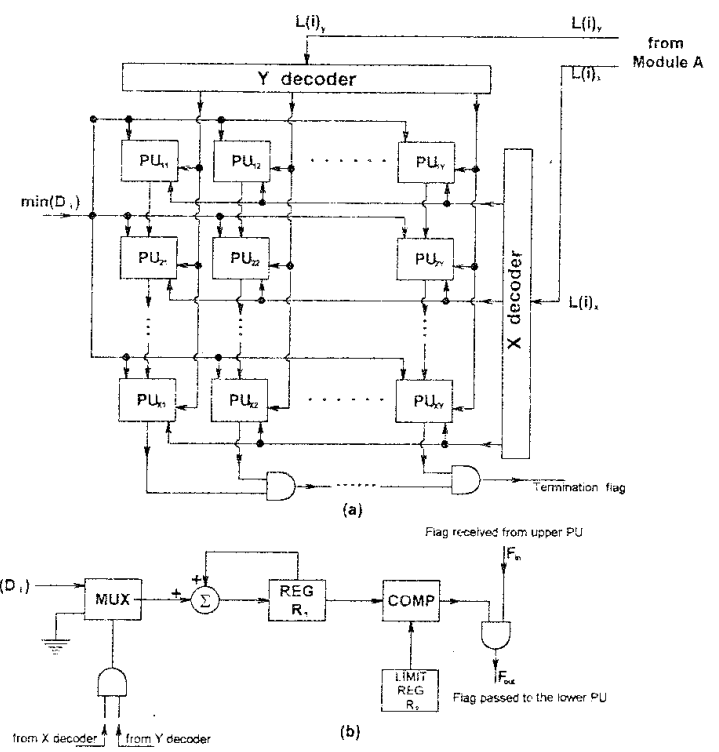

Fig. 7 Architecture for Module D

(a) System organization (b) Internal structure of PU

\section{PERFORMANCE EVALUATION}

In this section, the computational and circuit complexities of the proposed architecture are evaluated. The results are summarized by a comparison of the proposed architecture against the architecture presented in [9].

The PEs and additional circuit elements used in Modules A, B, $\mathrm{C}$, and $\mathrm{D}$ of the proposed architecture and the architecture in [9] are tabulated in Table 1.

Since $N$ is normally much greater than $M$ and $K$ in clustering analysis, in contrast to [9], the proposed architecture can save a tremendous amount of hardware for Modules A, C, and D in VLSI implementation. For a typical example of a codebook design for vector quantization, if $M=16$ and a codebook size $K=256$ with a frame of $256 \times 256$ image is employed, then $N=\frac{256 \times 256}{16}=4,096$ will be required. For Module A, only 4,096 PEs are used in the proposed architecture in comparison with one million $\left(=2^{20}\right)$ PEs required by the architecture in [9]. For Module $C$, only 888 
registers are used while 73,726 registers are demanded in [9]. For Module D, only 256 PEs are employed instead of 4,096 PEs in [9].

Table 1 Summary of circuit complexity (for $\mathrm{X}=\mathrm{Y}=\sqrt{K}$ )

\begin{tabular}{|l|l|l|}
\hline & Proposed architecture & Architecture in [9] \\
\hline Module A & $\begin{array}{l}M \mathrm{x} K \text { PEs } \\
(\sqrt{K}-1) \text { cell } \mathrm{C}_{1}\end{array}$ & $\begin{array}{l}N \mathrm{x} K \text { PEs } \\
N-1) D \text { registers } \\
N \text { buffers }\end{array}$ \\
\hline Module B & $\begin{array}{l}M \mathrm{x} K \text { PEs } \\
2 \text { decoders } \\
K \text { counters } \\
K \text { 2-input AND gates }\end{array}$ & $\begin{array}{l}M \times K \text { PEs } \\
(K-1) \text { registers } \\
1 \text { decoder }\end{array}$ \\
\hline Module C & $M\left(\frac{3}{2} M+2 \sqrt{K}-\frac{1}{2}\right)$ & $N(M+2)-2$ \\
& registers \\
& registers & \\
\hline Module D & $K$ PEs & $N$ PEs \\
& 2 decoders & $(N-1)$ registers \\
& $(\sqrt{K}-1)$ 2-input AND & 2-input AND \\
& gates \\
\hline
\end{tabular}

The computational complexity of the proposed architecture compared with the architecture in [9] is listed in Table 2.

The execution time for one pass operation of the proposed architecture is $N+M+3 \sqrt{K}+1$ clock cycles. In contrast to the $N+M+K+3$ time units required in [9], the proposed architecture can reduce $(K-3 \sqrt{K})+2$ time units. For the same example of a codebook design of $K=256$ as described above, 210 clock cycles can be saved for one pass operation.

Table 2 Summary of computational complexity (for $\mathrm{X}=\mathrm{Y}=\sqrt{K}$ )

\begin{tabular}{|l|l|c|}
\hline & $\begin{array}{l}\text { Proposed } \\
\text { architecture }\end{array}$ & Architecture in [9] \\
\hline Module A & $N+M+2 \sqrt{K}-1$ & $N+M+K+1$ \\
\hline Module B & $N+M+3 \sqrt{K}+1$ & $N+M+K+3$ \\
\hline Module C & $N+M+3 \sqrt{K}+1$ & $N+M+K+3$ \\
\hline Module D & $N+M+2 \sqrt{K}+1$ & $N+M+K+2$ \\
\hline
\end{tabular}

\section{CONCLUSIONS}

A novel systolic architecture for the squared-error clustering algorithm has been proposed. The architecture employs intensively parallel and pipelined processing techniques to reduce execution time. In addition, the organization based on 2-dimensional arrays $(K=X \times Y)$ further speeds up the computation. If $K$ is the square of an integer number, the one pass time complexity of the proposed architecture has been found to be $N+M+3 \sqrt{K}+1$, in comparison with the results of $N+M+K+3$ or $N \operatorname{xmax}(M, K)$ in previous architectures. For a large $K$, the reduction in execution time using the proposed architecture is significant.

The proposed architecture employs a 2-dimensional decoding technique in Module B and Module D. In comparison with the 1- dimensional linear decoding, the 2-dimensional decoding can save a lot of hardware in decoder. This advantage is notable in particular when $\mathrm{K}$ is large.

For clustering analysis applications in image processing, the number of input patterns, $N$, may vary in most situation. It is observed that the organizations of Module A, B, C, D of the proposed architecture are all independent of $N$. This characteristic makes the proposed architecture suitable for applications with various $N$ values.

\section{REFERENCES}

[1] R. C. Dubes and A. K. Jain, "Clustering methodologies in exploratory data analysis ", Advances in Computers, vol. 19, pp. $113 \sim 228$, Academic, 1980.

[2] G. B. Coleman and H. C. Andrew, "Image segmentation by clustering ", Proc. IEEE, vol. 67, pp. $773 \sim 786$, May 1979.

[3] G. Stockman, S. Kopstein, and S. Benett, "Matching images to models for registration and object detection via clustering, " IEEE Trans. Pattern Analy. Machine Intell., vol. PAMI-4, pp $229 \sim 241$, May 1982.

[4] J. Bryant, "On the clustering of multidimensional pictorial data," Pattern Recognition, vol. 11, pp. 115 125, 1979.

[5] W. H. Equitz, "A new vector quantization clustering algorithm", IEEE Trans. Acout. Speech, Signal Processing, vol. 37 , no. 10 , pp. $15 \epsilon 8 \sim 1575$, Oct. 1989.

[6] R. C. Dubes and A. K. Jain, "Clustering techniques: The user's dilemma", Pattern Recognition, vol. 8, pp. 247 260, 1976.

[7] L. M. Ni and A. K. Jain, "A VLSI systolic architecture for pattern clustering, "IEEE Trans. Pattern Analy. March, Intell., Vol. PAMI-7, pp. 80-89, Jan. 1985.

[8] H. D. Cheng and C. Tong, "Clustering analysis for pattern recognition", Proc. SPIE Visual Communication and Image Processing, Cambridge, MA, Oct. 1989.

[9] H. D. Cheng and C. Tong, " Clustering Analyzer, "IEEE Trans. Circuits and Systems, vol. 38, no. 1, pp. 124 128, Jan. 1991.

[10] J. T. Tou and R. C. Gonzalez, Pattern Recognition Principles, Addison-Wesley publishing Company, London, 1974.

[11] G. A. Davidson, P. R. Cappello, and A. Gersho, "Systolic architecture for vector quantization", IEEE Trans. Acout. Speech, Signal Processing, vol. 36, no. 10, pp. $1651 \sim 1664$, Oct. 1988.

[12] H. T. Kung, "Why Systolic Architecture?", Computer, vol. 15, no. 1, pp.37 45, Jan. 1982

[13] A. Gersho and V. Cuperman, "Vector quantization: A patternmatching technique for speech coding, "IEEE Commun. Mag., vol. 21, pp. $15 \sim 21$, Dec. 1983.

[14] S. Y. Kung, VLSI Array Processors, Prentice-Hall Inc., New Jersey, 1988.

[15] C. H. Hsieh and T. P. Lin, "VLSI Architecture for Blockmatching Motion Estimation Algorithm", IEEE Trans. Circuits and Systems for Video Technology, vol. 2, no. 2, pp.169 175, June 1992. 